\title{
Moderna COVID-19 vaccine
}

Vaccine Ontology (VO)

\section{Source}

Vaccine Ontology (VO). Moderna COVID-19 vaccine. VO:0005157.

A SARS-CoV2 RNA vaccine made of lipid nanoparticle with mRNA which encodes the S-2P antigen, made of the SARS-CoV-2 glycoprotein with a transmembrane anchor and intact S1-S2 cleavage site. 